\title{
Representaciones sociales sobre medicina tradicional y enfermedades foráneas en indígenas EmberaChamí de Colombia
}

\section{Social representations about traditional medicine and foreign diseases in EmberaChami natives in Colombia}

\author{
MSc. Jaiberth Antonio Cardona Arias, Dra. Yennifer Rivera Palomino \\ Universidad de Antioquia, Escuela de Microbiología. Medellín, Departamento de \\ Antioquia, Colombia.
}

\section{RESUMEN}

Introducción: el fomento de la interculturalidad en salud ha sido obstaculizado por el desconocimiento de las particularidades de los sistemas médicos indígenas. Las enfermedades foráneas constituyen uno de los casos en que se demuestra la necesidad de articular la medicina tradicional con la occidental.

Objetivo: comprender las representaciones sociales sobre medicina tradicional y algunas enfermedades foráneas en indígenas EmberaChamí de Colombia.

Métodos: mediante la técnica de muestreo se seleccionaron 22 indígenas. Se realizaron entrevistas para codificación abierta, axial y selectiva, diarios de campo y observación participante y no participante. Se siguieron los criterios de credibilidad, auditabilidad, transferibilidad y saturación de categorías. La recolección y el análisis de la información se desarrollaron entre agosto de 2010 y julio de 2011 . Se observaron los principios éticos para la investigación en salud.

Resultados: se presentan representaciones sociales sobre el sistema médico tradicional EmberaChamí y enfermedades foráneas, se construyó una matriz de significación fundamentada en la Pacha Mama y los constructos de Cosmogonía, Cosmovisión y Teoría de los tres mundos. El sistema médico tradicional está compuesto por el acervo cultural, el territorio y soberanía, y los recursos terapéuticos. El proceso salud enfermedad es la armonía en las dimensiones psíquico mental, espiritual, social, ambiental y físico-biológica, en esta última se circunscriben las concepciones y manejo de las enfermedades foráneas.

Conclusión: se abordan algunos aspectos del universo simbólico indígena que potencian la interculturalidad en salud, fomentan el abordaje holístico de las enfermedades foráneas y posibilitan el uso contextualizado del saber ancestral con base en las cosmologías, estructura social, ritos y otras especificidades de las comunidades EmberaChamí.

Palabras clave: Medicina tradicional, enfermedades, indígenas, Colombia. 


\section{ABSTRACT}

Introduction: the promotion of interculturalism in health has been hampered by ignorance of the particularities of indigenous medical systems. Foreign diseases are one of the cases which show the need of integrating the traditional and the Western medicine.

Objective: to understand the social representations of traditional medicine and foreign diseases in EmberaChami natives in Colombia, 2011.

Methods: twenty two natives were selected through sampling. Interviews were made for open, axial and selective coding, field records and participant and nonparticipant observation. The criteria of credibility, auditability, transferability and saturation of categories were applied. Data was gathered and analyzed from August 2010 to July 2011 . The ethical principles for research in the health field were respected.

Results: social representations about the traditional medical system EmberaChami and foreign diseases were presented; a matrix of significance based on the Pacha Mama and Cosmogony, Cosmovision and Theory of the three worlds was prepared as well. The traditional medical system consists of the cultural heritage, the territory and sovereignty, and the therapeutic resources. The health-disease process is conceived as the harmony of the psychical, mental, spiritual, social, environmental and physical-biological dimensions. The latter comprises the conceptions and management of foreign diseases.

Conclusion: this study allowed us to address aspects of the native symbolic universe that enhance intercultural health, promote the holistic approach of foreign diseases and enable the contextualized use of traditional knowledge based on the cosmology, social structure and rituals of the EmberaChami communities.

Key words: Traditional medicine, diseases, natives, Colombia.

\section{NTRODUCCI ÓN}

El Sistema de Salud Colombiano, reformado en 1993, presentó como objetivo prioritario garantizar la prestación de servicios de salud a través del aseguramiento universal y la superación de las barreras de acceso, no obstante, en el ámbito nacional persisten múltiples problemas, ${ }^{1}$ que para los pueblos indígenas han sido básicamente el transporte, la ubicación geográfica y las limitaciones económicas. ${ }^{2}$ Estos obstáculos, sumado a la baja confianza en la medicina occidental y las falencias del modelo de aseguramiento en salud, han incrementado el uso de la medicina tradicional indígena. ${ }^{3}$

La medicina tradicional indígena es la suma de conocimientos, tradiciones, prácticas y creencias de una cultura, utilizados para mantener la salud y prevenir, diagnosticar o tratar la enfermedad, a partir de un sistema que presenta roles y recursos terapéuticos propios. ${ }^{4,5}$ Su uso se ha reglamentado en más de 100 países que han comprobado su relevancia, principalmente en comunidades rurales, ${ }^{4}$ no obstante, su articulación con sistemas de salud institucionales no ha sido eficiente, ${ }^{6}$ lo que constituye un gran reto para lograr la interculturalidad en salud. 
A pesar de las iniciativas de la Organización Mundial de la Salud (OMS) por potenciar la interculturalidad en salud, esta es exigua en Colombia, lo que representa un problema de salud para los pueblos indígenas por las siguientes razones: 1. En estas comunidades ha aumentado la frecuencia de enfermedades foráneas como la diabetes, ${ }^{7}$ la hipertensión arterial y las dislipidemias, para las cuales, el sistema médico tradicional no tiene opciones terapéuticas; 2 . Las acciones en salud implementadas por instituciones oficiales en los resguardos generalmente son verticales, ya que no incluyen el cúmulo de conocimientos y experiencias de su cultura, lo que deriva en un bajo impacto sobre la salud individual y colectiva, y 3 . Sectores oficiales creen que las comunidades indígenas son un grupo homogéneo y desconocen las diferencias en sus dialectos, modos de vida, procesos productivos, ${ }^{8}$ mitos fundantes, visión de mundo y en los componentes empíricos inherentes al abordaje del proceso salud enfermedad, lo que en el caso colombiano implica un error craso dado que en este país existen 102 pueblos indígenas con 1378884 personas, distribuidos en 710 resguardos con cosmologías diversas. ${ }^{9}$

En concordancia con lo anterior, se realizó un estudio con el objetivo de comprender las representaciones sociales sobre medicina tradicional y algunas enfermedades foráneas en indígenas EmberaChamí de Colombia, como elemento central para fomentar la interculturalidad en salud y la identidad étnica a partir de la conservación y reivindicación de los saberes acumulados por este grupo. La relevancia de comprender las representaciones sociales radica en que estas integran lo individual y lo colectivo, lo simbólico y lo social, el pensamiento y la acción; subsumen experiencias, informaciones y modelos de pensamiento que permiten aprehender la realidad, reconocer procesos de constitución del pensamiento social, aproximarse a la visión de mundo de las personas, comprender las conductas sociales e interpretar la comunicación entre los miembros de una comunidad. ${ }^{10-12}$

El estudio se realizó con indígenas Embera, uno de los 102 pueblos indígenas de Colombia, con algunas comunidades en Panamá y Ecuador; estos presentan cuatro formas dialectales EmberaDobidá, EmberaChamí, EmberaKatío y SíaPedeé; según el medio en que viven se dividen en Eyábida (hombres de montaña) y Dobidá (hombres de río), el pueblo EmberaChamí pertenece al primer grupo, tiene la mayor dispersión territorial en Colombia y está cohesionado por la lengua yla relación mágicoreligiosa con los espíritus de la naturaleza y la relación con el territorio. $^{8}$

\section{MÉTODOS}

Investigación cualitativa desarrollada con 22 adultos de etnia EmberaChamí de Colombia, se incluyeron 5 médicos tradicionales, 2 parteras, 3 curanderos, 1 rezandero, el coordinador del Programa de Defensa de la Salud Integral Indígena, 2 promotoras de salud, 2 auxiliares de enfermería, 2 estudiantes de una Escuela de Médicos Tradicionales y 4 comuneros, seleccionados por muestreo.

La recolección y el análisis de la información se desarrollaron entre agosto de 2010 y julio de 2011, a través de entrevistas semiestructuradas grabadas y transcritas en su totalidad, diarios de campo, observación no participante y participante, realizadas con el fin de registrar comportamientos, cotidianidades e interacciones entre los comuneros. A cada participante se le realizaron tres entrevistas correspondientes a la codificación abierta donde se conformaron las categorías descriptivas, codificación axial para establecer relaciones entre las categorías 
descriptivas, definir las analíticas e identificar las categorías y sub-categorías, y codificación selectiva para determinar la categoría central de la matriz de significación (recuadro). ${ }^{13}$

Recuadro. Sistema categorial que configuró la matriz de significación

\begin{tabular}{|c|c|c|}
\hline Categoría & Subcategorías & Tipo \\
\hline \multirow{3}{*}{$\begin{array}{l}\text { Conceptos } \\
\text { conexos al } \\
\text { cosmos }\end{array}$} & Cosmogonía & Emergente \\
\hline & Teoría de los tres mundos & Emergente \\
\hline & Cosmovisión & Emergente \\
\hline \multirow{3}{*}{$\begin{array}{l}\text { Medicina } \\
\text { Tradicional } \\
\text { Indígena }\end{array}$} & Algunas consideraciones conceptuales & Preestablecida \\
\hline & Escuela de Médicos Tradicionales & Emergente \\
\hline & Proceso Salud-Enfermedad-Atención & Preestablecida \\
\hline \multirow{3}{*}{$\begin{array}{l}\text { Enfermedades } \\
\text { foráneas }\end{array}$} & Teoría de causalidad de la enfermedad & Preestablecida \\
\hline & Conceptualización & Preestablecida \\
\hline & $\begin{array}{l}\text { Concepción y manejo tradicional de algunas } \\
\text { enfermedades foráneas }\end{array}$ & Emergente \\
\hline
\end{tabular}

Las representaciones sociales se abordaron desde el enfoque procesual, el que posibilita la recolección de material discursivo generado de manera espontánea por el participante, para luego realizar análisis hermenéutico. Se ejecutó análisis comparativo constante, el cual es útil para abordar las representaciones sociales, dado que permite una aproximación descriptiva del contenido del discurso y una relacional explicativa para develar estructuras internas de la narrativa del sujeto. ${ }^{10}$

\section{CRITERIOS DE RIGOR METODOLÓGICO}

Credibilidad, auditabilidad, transferibilidad, saturación de categorías y triangulación investigativa, metodológica y teórica. ${ }^{14}$

\section{ASPECTOS ÉTICOS}

Se siguieron los principios de la resolución 8430 de 1993 del Ministerio de Salud de Colombia por la cual se establecen las normas científicas, técnicas y administrativas para la investigación en salud. Se contó con el consentimiento colectivo de la autoridad indígena e individual para cada participante.

\section{RESULTADOS}

Los participantes pertenecen a un resguardo ubicado en el departamento de Caldas, que tiene una población de 21892 habitantes. La primera autoridad es el Gobernador Principal acompañado del Gobernador Suplente, seguidos de alcalde primero y segundo, regidor primero y segundo, alguacil primero y segundo, 33 cabildantes y 4 áreas que agrupan el trabajo comunitario: Administración y Gestión, Justicia Propia, Territorio y Autonomía, y Cultura y multiculturalidad, esta 
última subsume los componentes de etnoeducación, deportes y recreación, mujer y familia, defensa de la salud integral indígena y asamblea de la comunidad.

El contenido teórico, mitológico y empírico que orienta la vida de las comunidades EmberaChamí está cohesionado por una concepción de cosmos cuyo eje es la Pacha Mama (madre naturaleza), dicha concepción se cristaliza en cosmogonías y cosmovisiones, términos que dotan de sentido la cotidianidad de los indígenas, su interacción con el entorno, con sus álter ego y con la divinidad.

\section{CONCEPTOS CONEXOS AL COSMOS}

Cosmogonía. Conjunto de teorías míticas, religiosas, filosóficas y científicas sobre el origen del mundo, es el eje del equilibrio entre los comuneros y de estos con los astros, por ello, la mayoría de los fenómenos de la naturaleza, incluyendo la salud y la enfermedad, son determinados por los cuerpos celestiales. Cosmogonía es estar totalmente identificado, en la profundidad del universo, con las estrellas, con la luna, con los astros... y la naturaleza indómita que no acepta normas.

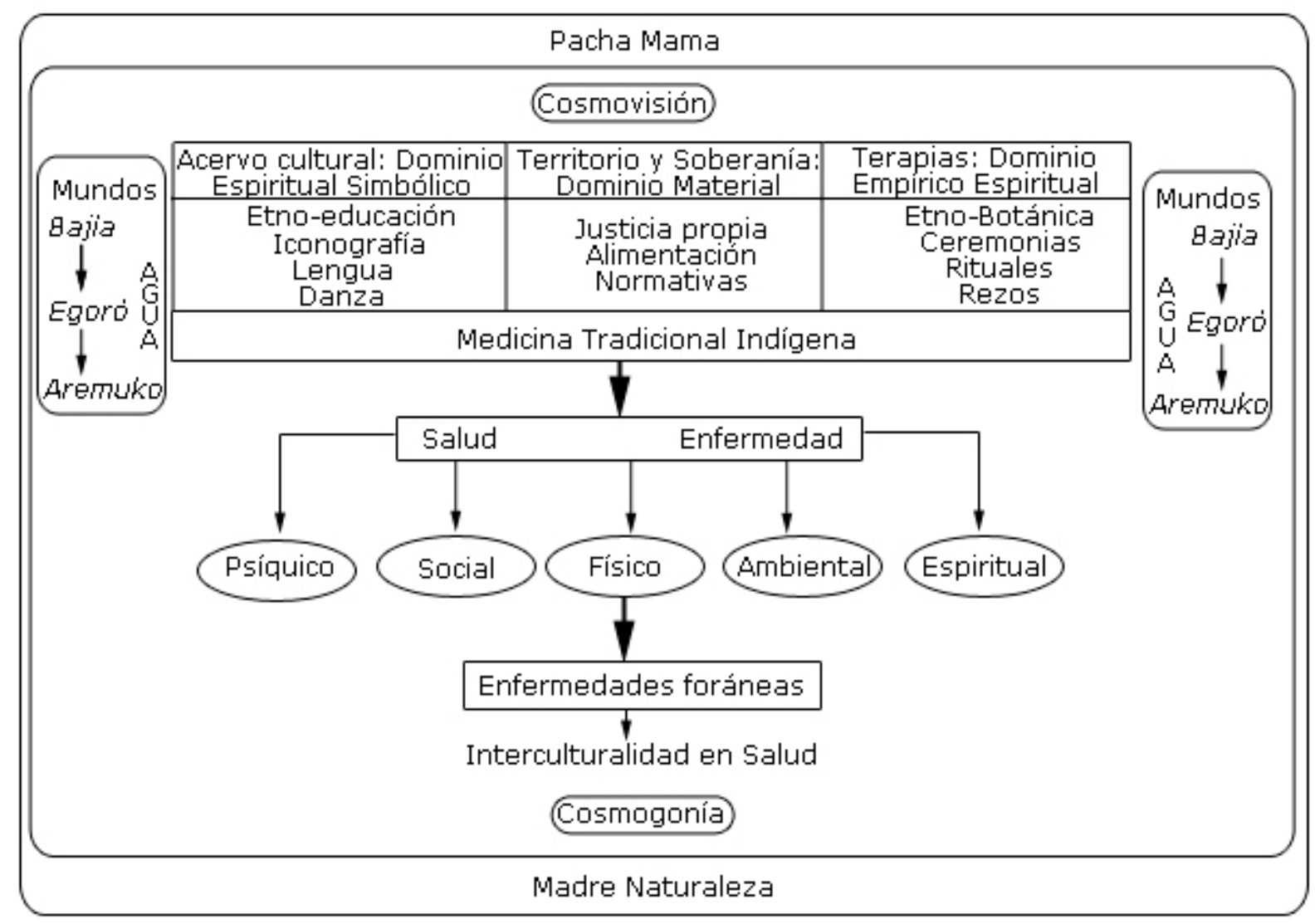

Fig. Matriz de significación sobre Medicina Tradicional Indígena y enfermedades foráneas en indigenas EmberaChami de Colombia.

Teoría de los tres mundos. Como parte de la cosmogonía, el pueblo EmberaChamí vive su cotidianidad con base en los principios de unidad, tierra, cultura y autonomía, y en la teoría del origen del mundo que se atribuye la configuración de tres componentes que separan lo material de lo espiritual; en primer lugar está bajía, término en lengua embera que significa mundo celestial o "de arriba", conformado por Karagabí (luna),Ba (trueno), sitios sagrados (como montañas, lagunas y cascadas), espíritus ancestrales y guardianes del territorio (espíritus 
representados en algunas elementos de la iconografía indígena o en forma de energías que circulan al interior de los sitios sagrados); luego está egoró, mundo medio o de los humanos, y en último término aremuko o chiapera, mundo subterráneo o "de abajo", donde convergen principalmente espíritus o energías negativas. Los tres mundos se conectan a través del agua, por ello este es un elemento sagrado.

Cosmovisión. Proyección del pensamiento propio a los diferentes elementos de la naturaleza, para visionar cada punto de entrada y salida del sentido espiritual; esta se ha constituido a partir de diálogos entre los mayores (personas del resguardo cuya espiritualidad y sabiduría les confiere autoridad al interior de la comunidad) que han agrupado múltiples conocimientos para comprender el universo (Fig.).

\section{MEDI CI NA TRADI CI ONAL I NDÍ GENA}

Consideraciones conceptuales. Parte del acervo cultural transmitido oralmente a través de múltiples generaciones y constituido por los saberes y prácticas relacionadas con la vida, la armonía, el equilibrio y el bienestar del pueblo indígena; ha sido utilizada por miles de años con base en los conocimientos que hacen parte de la ancestría indígena. La Pacha Mama es la encargada de brindar, a través de las plantas y los elementos de la naturaleza, el saber a los médicos para que se pueda dar la sanación de una enfermedad; en este sentido, el médico tradicional solo actúa como intermediario entre la madre naturaleza y el paciente, al "activar la relación con la madre naturaleza."

Escuela de médicos tradicionales. Dada la pérdida de los conocimientos tradicionales por procesos de mestizaje y por el deseo de muchos indígenas jóvenes de migrar a las ciudades, las comunidades indígenas han implementado diversas estrategias para enfrentar este problema, como la sistematización de saberes propios, la edición de algunos textos, el fomento del arraigo cultural en la educación básica y media, y la creación de escuelas de médicos tradicionales. La escuela de médicos tradicionales es la encargada de formar a las nuevas generaciones en los dominios espiritual, físico, social y ambiental de la medicina tradicional; la escuela tiene un plan de formación de aproximadamente 5 años en el que pueden participar comuneros del resguardo que hayan culminado los estudios de educación básica y media y hayan trabajado en el área de salud del resguardo. En los primeros años se aborda el estudio del cuerpo humano y la etnobótanica, en los años siguientes se hace un trabajo de ascenso espiritual donde se valora la capacidad del estudiante para interactuar con los espíritus y los guardianes del territorio, para canalizar la energía de los sitios sagrados y en general para intermediar en la relación de las personas con la Pacha Mama:

Nuestros mayores se nos están yendo con el conocimiento y no lo están dejando plasmado, la idea es tener una escuela para que ese conocimiento quede en memorias y que el conocimiento que hoy tengo lo puedan aprovechar mis hijos, mis nietos y así sucesivamente. Se ha creado una escuela llamada escuela de médicos tradicionales "Valle de los Pirzas", donde está plasmado el conocimiento de los mayores en todo lo que tiene que ver con el fortalecimiento del saber natural en la medicina y de los procesos políticos organizativos. El proceso dura 5 años, estudiamos domingos y lunes, incluyendo todas las noches para lo que tiene que ver con la espiritualidad. Nos han enseñado el proceso de diagnóstico, nos han dado clases de anatomía y fisiología, hemos salido a conocer algunas de las plantas de nuestro territorio, nos han enseñado a hacer cura de ojo, nos han enseñado la oración del tabaco y otras cosas. 
Proceso salud enfermedad atención. La salud es conceptualizada desde una perspectiva holística como la armonía entre los dominios físico, psíquico, social, espiritual y ambiental, lo que depende de la relación entre el hombre y la madre naturaleza, esta es quien posibilita la salud y el equilibrio de energías al interior de los resguardos. "Salud es el estado de armonía entre cuerpo, mente y espíritu; cuerpo es lo físico, mente son los pensamientos y espíritu lo relacionado con los elementales de las plantas y el territorio... para que haya un buen equilibrio dentro de nuestra sociedad, debe haber buen equilibrio en nuestro cuerpo, nuestra mente y nuestro espíritu."

En el abordaje del proceso salud-enfermedad se diferencia la sanación y la curación, la primera alude el manejo físico y espiritual de la enfermedad a través de la etnobotánica (uso de las propiedades curativas de las plantas y las energías o espíritus que fluyen a través de ellas) y la intermediación del médico tradicional como agente que restablece las relaciones del hombre con el mundo espiritual, mientras que curar alude a un trabajo superfluo o paliativo donde se aborda básicamente el componente físico sin restablecer la armonía espiritual, la curación es un término tomado de la medicina occidental referido a eliminación de dolencias.

En concordancia con la noción de sanación, la mayoría de las enfermedades son tratadas a través de rezos y procesos que involucran el equilibrio de energías, para algunas sanaciones se hace necesario estar en un sitio sagrado.

Aunado a lo anterior, en el resguardo existen los guardianes o espíritus de los ancestros que controlan las energías, se comunican con los médicos para acompañarlos y darles la sabiduría y espiritualidad requerida para sanar las enfermedades; estos se encuentran en los sitios sagrados en forma de energía y en el territorio en forma de íconos que han sido enterrados por los antepasados, y son invocados cuando se encuentran desequilibrios al interior de la comunidad.

\section{ENFERMEDADES FORÁNEAS}

Teoría de causalidad de las enfermedades. En concordancia con la teoría de los tres mundos y de la concepción de salud, en el acervo cultural indígena, la enfermedad obedece a un desequilibrio en las dimensiones física, psíquica, espiritual, social o ambiental, el cual puede atribuirse a tres causas diferentes: 1. Al desequilibrio con las energías positivas o al distanciamiento del indígena de su vida espiritual o lejanía de bajía (mundo celestial), 2. Al contacto con energías negativas o espíritus malignos y 3 . Al mestizaje.

Los dos primeros son considerados causales de enfermedades propias para las cuales el sistema médico tradicional presenta múltiples opciones terapéuticas fundamentadas en etnobotánica, rezos, armonizaciones, rituales y ceremonias; por su parte, el tercer grupo, obedece a enfermedades foráneas, para los cuales la medicina tradicional presenta pocas opciones terapéuticas.

Conceptualización. Las enfermedades foráneas son desequilibrios o alteraciones físico biológicas, propias de la "raza blanca", cuya presentación en los indígenas obedece a procesos de mestizaje donde el indígena adopta costumbres del mundo occidental, principalmente en el componente alimentario.

En la práctica tradicional existen recursos terapéuticos para las enfermedades propias basados en el equilibrio de energías en los ámbitos individual, social y 
natural, no obstante, las foráneas traspasan el cúmulo de conocimientos tradicionales y sus opciones terapéuticas, lo que evidencia la necesidad de interactuar con otras prácticas médicas y de potenciar la etnoeducación.

Entre las principales enfermedades foráneas identificadas por los miembros del Área de Defensa de Salud Integral Indígena, se identifican la artritis, reumatismo, tensión arterial alta, diabetes mellitus, dislipidemias, anemia y parasitismo intestinal. Algunos médicos tradicionales aluden a estas alteraciones con la misma nominación que en el sistema médico occidental, lo cual es explicado por un proceso de migración conceptual, donde los indígenas incorporan conceptos del mundo occidental por la interacción con comunidades campesinas o los hospitales y por las campañas de educación en salud que desarrollan las instituciones oficiales.

Concepciones y manejo tradicional de enfermedades foráneas. Entre ellas:

- Dislipidemias: en la cultura EmberaChamí no existe esta denominación, al igual que en las comunidades rurales campesinas, esta entidad se identifica con alteraciones del colesterol bueno y malo y de los triglicéridos, términos comunes para la mayoría de personas que han recibido atención en hospitales. Esta alteración se atribuye al aumento en el consumo de productos occidentales, a la influencia que ha tenido la lógica de mercado y el consumismo en las generaciones jóvenes de indígenas; todo ello, como consecuencia de la pérdida de la identidad cultural. Para su diagnóstico, los médicos tradicionales emplean la técnica de visualización de orina y en algunos casos solicitan los exámenes propios de la medicina occidental mientras que en el tratamiento se emplean plantas como masequia, guineo, chaparro, cola de caballo, caracola y suelda redonda, en forma de infusiones o brebaje.

- Diabetes mellitus: de forma similar a las dislipidemias, la diabetes o azúcar en la sangre se explica por la pérdida de identidad cultural en el componente alimentario y por antecedentes familiares, su diagnóstico se realiza a través de la orina y las principales plantas para tratarla son casco de buey, chaparro y yacón.

La diabetes se produce en algunas ocasiones por herencia, por estrés, por el consumo de muchos dulces o porque el organismo no produce insulina; la idea es que las plantas ayuden a regular el azúcar o la insulina. Para los médicos tradicionales es muy complicado diagnosticar el azúcar en la sangre, nos basamos primero porque al paciente le da mucha sed, cuando una persona se corta y la herida se demora mucho para sanar, la orina se pone de un color casi cristalino, cuando la persona orina demasiado.

- Anemia y parasitismo intestinal: nosotros la anemia la catalogamos como la falta de hemoglobina, es producida por parásitos o gusanos que chupan la sangre, pero también por una mala alimentación o la picadura de algún vector como el del paludismo. Cuando hay una sobrecarga de parásitos, esto hace que los parásitos sean quienes se consuman el alimento y obviamente las defensas nuestras van a bajar, y una vez estén afectadas nuestras defensas empieza a haber entonces el desequilibrio y ya puede haber la aparición de una anemia. Al interior del sistema médico, las principales opciones terapéuticas para la anemia son boldo, ajo, yerbabuena, ajenjo y lombricera cuando es de origen parasitario, si es de tipo nutricional se recomienda el aumento en el consumo de zapallo, guineo, plátano mataindio, chengue o meme, vino macerado de romero, hígado, pajarilla y mora. 


\section{DISCUSIÓN}

En el estudio se presentaron algunas de las representaciones sociales relacionadas con el sistema médico tradicional EmberaChamí y enfermedades foráneas, con base en las cuales se construyó una matriz de significación fundamentada en la Pacha Mama, los constructos de Cosmogonía, Cosmovisión y Teoría de los tres mundos, y los tres ámbitos del sistema médico tradicional: acervo cultural, territorio y soberanía, y recursos terapéuticos. En este pueblo la causalidad de las enfermedades está determinada por un componente espiritual energético y otro físico, en este último se ubican las enfermedades foráneas como la dislipidemia, diabetes y anemia, cuyo diagnóstico, tratamiento, seguimiento, control y prevención demuestra la necesidad de la interculturalidad en salud, debido a las limitaciones que presenta el Sistema Médico Indígena para abordarlas.

La interculturalidad en salud es coherente con normativas internacionales y nacionales que explicitan la necesidad de enfoques holístico de salud, de la reciprocidad entre actores y recursos terapéuticos de la medicina occidental y la tradicional indígena, y la incorporación de la medicina tradicional a los sistemas nacionales de salud. ${ }^{15-21}$ Concretamente en Colombia, la Ley 691/2001 busca garantizar la integridad y permanencia cultural de la salud indígena, y fundamentar la prestación de servicios de salud con base en los principios de pluralismo médico, complementariedad terapéutica e interculturalidad. ${ }^{21}$

La etnia EmberaChamí, al igual que otros indígenas, presenta una concepción de mundo materializada en cosmovisiones y cosmogonías donde priman las relaciones con la madre naturaleza y los seres espirituales, y se configura la identidad cultural y la permanencia de saberes propios, dichos constructos agrupan los pensamientos, creencias y prácticas indígenas que buscan la armonía, la integralidad y la cohesión de procesos ideológicos, políticos, espirituales y empíricos. ${ }^{6,22}$

Como parte de la cosmovisión y cosmogonía, la medicina tradicional indígena, en las comunidades EmberaChamí y otras referidas en diversos estudios, cumple un rol cultural central para la explicación de múltiples fenómenos de la cotidianidad, para lograr la armonía en el territorio y agrupar parte del arraigo histórico y sociocultural; sus conocimientos y prácticas son integrados culturalmente y transmitidos de generación en generación, teniendo como principal recurso terapéutico a la Etnobotánica. ${ }^{22,23}$ En este tipo de sistema médico, la medicina tradicional es reconocida, sustentada, resguardada y realimentada por la comunidad, ya que esta tiene un rol protagónico en las relaciones que se establecen con la naturaleza y la divinidad. ${ }^{24}$

La conceptualización del proceso salud enfermedad, en esta y otras comunidades indígenas, se caracteriza por su holismo, donde la enfermedad es resultado del desequilibrio en las relaciones del ser humano con sus semejantes, su ambiente y sus deidades. ${ }^{24-26}$ La teoría de la causalidad de las enfermedades subsume la espiritualidad y el dominio físico biológico, esto congruente con lo registrado en otros pueblos donde la enfermedad se atribuye a malestar enviado por Dios, encantos, vientos y espíritus que actúan de forma espontánea o son dirigidos por otras personas que quieren generar daño. 27,28

Las opciones terapéuticas de la medicina EmberaChamí resultan similares a las informadas en otros estudios, al agrupar prácticas para estabilizar las energías que producen daño en el espíritu y plantas medicinales preparadas en ungüentos, pomadas, infusiones, bebidas, maceraciones y emplastos, cuyo éxito depende de la fe de las personas que las utilizan; ${ }^{25}$ dichos recursos resultan limitados para el 
diagnóstico, manejo y prevención de las enfermedades foráneas, afianzando la necesidad de la interculturalidad en salud.

El abordaje de las representaciones sociales permite comprender los constructos que orientan las acciones, los sentidos y los significados que las personas le atribuyen a los objetos, situaciones y fenómenos de su cotidianidad como resultado de la conjunción de lo individual y lo colectivo. ${ }^{29,30} \mathrm{Su}$ conocimiento posibilita un mayor éxito en los programas en salud dado que las acciones sanitarias presentan mayores beneficios cuando introducen aspectos socioculturales, experiencias y saberes acumulados por los actores sociales, cuando buscan la integración social, emplean formas discursivas próximas al lenguaje de la comunidad.

La teoría de los 3 mundos que determinan la cotidianidad de la etnia EmberaChamí incluyendo su sistema médico, demuestra la relevancia del dominio espiritual y del agua. Como parte de esta teoría, el mundo de los humanos se subdivide en tres ámbitos: el monte o selva, las orillas de los ríos que constituyen la tierra habitada por los Embera y el agua de los ríos; el monte es donde se ubican los nacimientos de agua y constituye un medio lleno de peligros, es la vía de comunicación con el "mundo de abajo", mientras que el río es el medio de interacción con el mundo celestial. $^{30}$

De esta comunidad se debe destacar el trabajo educativo de la escuela de médicos tradicionales y su plan de formación de cinco años avalado por el Ministerio de Educación, y la concepción y manejo de algunas enfermedades foráneas, donde se presenta una articulación con el sistema médico occidental en la parte diagnóstica más no en la terapéutica, ya que es frecuente la remisión de los pacientes por parte del médico tradicional a los laboratorios clínicos para realización de glicemia, perfil lipídico, hemograma y otros exámenes, aunque en el tratamiento persiste hermetismo dado que este se fundamente casi exclusivamente en la etnobotánica.

Finalmente, este estudio permitió aludir a algunos aspectos del universo simbólico de los EmberaChamí que potencian la interculturalidad en salud, principalmente en el abordaje de enfermedades foráneas, y fomentan el uso contextualizado del saber indígena según sus cosmologías, estructura social, ritos y otras especificidades.

\section{Agradecimientos}

A las autoridades indígenas, los participantes y a la Universidad de Antioquia.

\section{REFERENCI AS BI BLI OGRÁFICAS}

1. Rodríguez S. Barreras y determinantes del acceso a los servicios de salud en Colombia. Universidad Autónoma de Barcelona. [Internet]. 2008 [citado 10 Nov 2011]. Disponible en: http://dep-economiaaplicada.uab.cat/secretaria/trebrecerca/Srodriguez.pdf

2. Vargas DL, Bañol ND. Análisis de la percepción y el grado de satisfacción en la relación médico paciente de la atención en medicina tradicional, Resguardo Indígena de San Lorenzo Riosucio Caldas. Facultad de Medicina, Universidad CES 
[Internet]. 2008 [citado 10 Nov 2011]. Disponible en http://bdigital.ces.edu.co:8080/dspace/bitstream/123456789/359/1/analisis_perce pcion_grado_satisfacion.pdf

3. Jorand B. Formas de transformación del conocimiento de la medicina tradicional en los pueblos nahuas del municipio de Hueyapan, Sierra Norte de Puebla. Rev Cuicuilco. 2008; 15(44):181-96.

4. Organización Mundial de la salud. Medicina tradicional [Internet]. 2008 [citado 10 Nov 2011]. Disponible en: http://www.who.int/mediacentre/factsheets/fs134/es/index.html

5. García MM E. Modelo de la Medicina indígena Maya en Guatemala. Investigación participativa en Sipacapa, San Marcos, San Martín Jilotepeque, Chimaltenango y San Jaunl xcoy, Huehuetenango. 2da ed. Guatemala: ASECSA-DED; 2003.

6. Hernández R, Amaya JA, Chávez MA. Promoción de la Medicina y Terapias Indígenas en la Atención Primaria de Salud: El caso de los Garífunas de Honduras. Washington, D.C.: OPS/OMS Honduras; 2002.

7. Asociación Latinoamericana de Diabetes. Guías ALaD de diagnóstico control y tratamiento de la diabetes Mellitus Tipo 2 [Internet]. 2006 [citado 10 Nov 2011]. Disponible en: http://www.fenadiabetes.org.ve/docs/guia.pdf

8. Asociación OREWA. Pueblo Indígena EmberaDobidá, Katio y Chamí [Internet]. 2010 [citado 15 Dic 2011]. Disponible en:

http: //orewa.org/index.php?option=com content\&view=article\&id=23: puebloindigena-embera-dobida-katio-y-chami\&catid=17: pueblos-indigenas\&l temid $=22$

9. ACNUR, Agencia de la ONU para los refugiados. Pueblos Indígenas en Colombia [Internet]. 2010 [citado 15 Dic 2011]. Disponible en:

http://www. acnur.org/t3/pueblos-indigenas/pueblos-indigenas-en-colombia/

10. Araya US. Las representaciones sociales: ejes teóricos para su discusión. Cuaderno de Ciencias Sociales. Costa Rica: Facultad Latinoamericana de Ciencias Sociales (FLACSO); 2002.

11. Moscovici S, Rosenbaum D. Psicología social II: pensamiento y vida social, psicología social y problemas sociales. Barcelona: Paidós; 1986.

12. Sandoval C. Sueños y sudores en la vida cotidiana de trabajadores y trabajadoras de la maquila y la construcción. San J osé, Costa Rica: Editorial de la Universidad de Costa Rica; 1997.

13. Galeano M. Estrategias de innovación social cualitativa. Medellín: La carreta editores; 2004.

14. Castillo E, Vásquez M. El rigor metodológico en la investigación cualitativa. Rev Colomb Med. 2003;34(3): 164-7. 
15. Organización Mundial de la Salud. Estrategia de la OMS sobre medicina tradicional 20022005 [Internet]. 2002 [citado 2 Dic 2011]. Disponible en:

http://apps.who.int/medicinedocs/fr/d/Js2299s/1.html

16. WüstZ F. A Medicina Tradicional e a Propriedade Intelectual. En: Ministério da saúde, Fundação Nacional de Saúde, editors. Saúde Indígena. Medicina tradicional indígena em contextos. Anais da i reunião de monitoramento. Brasilia, D.F.:

Tiragem; 2007. p. 132-44.

17. Organización Mundial de la Salud, Organización Panamericana de la Salud, División de Desarrollo de Sistemas y Servicios de Salud. Fortalecimiento y desarrollo de los sistemas de salud tradicionales: Organización y provisión de servicios de salud en poblaciones multiculturales. Washington D.C: División; 1997.

18. Morón F, Jardines B. La medicina tradicional en las universidades médicas. Rev Cubana Plant Med. 1997;2(1):35-41.

19. Padrón Cáceres L, Pérez Viñas M. Integración de las prácticas de la medicina tradicional y natural al sistema de salud [Internet]. 2007 [citado 15 Mar 2011]. Disponible en: http://www.undp.org.cu/pdhl/ideass/BrochureMTNesp.pdf

20. Pastrana AA, Santos JM, Ordóñez S. Ley 691 de 2001. Mediante la cual se reglamenta la participación de los Grupos Étnicos en el Sistema General de Seguridad Social en Colombia. Bogotá, D. C.: Gobierno Nacional; 2001.

21. López Hernández JR, Teodoro Méndez J M. La cosmovisión indígena Tzotzil y Tzeltal a través de la relación salud-enfermedad en el contexto de la medicina tradicional indígena. Rev Ra Ximhai. 2006;2(1):15-26.

22. Granados SM, Martínez LE, Morales P, Ortiz GR, Sandoval Hernando, Zuluaga G. Aproximación a la medicina tradicional Colombiana. Una mirada al margen de la cultura occidental. Revista Ciencias Salud Universidad Rosario. 2005;3(1): 98-106.

23. Secretaría de Salud, Estados Unidos mexicanos. Interculturalidad en Salud. Experiencias y aportes para el fortalecimiento de los servicios de salud. Indígenas [Internet]. 2008. [citado 10 Nov 2011]. Disponible en:

http://www. salud.gob. mx/unidades/cie/cms cpe/descargas

intercultural/Libro\% 20Interculturalidad\%20en\%20Salud\%2020.pdf

24. Instituto Interamericano de Derechos Humanos, Organización Panamericana de la Salud. Medicina indígena tradicional y medicina convencional. Módulo. San José, Costa Rica: Editorial IIDH, OPS; 2006.

25. Gutiérrez V. Tipología de la medicina tradicional y caracterización de los sistemas médicos. En: Gutiérrez de Pineda V. editor. Medicina tradicional de Colombia. Magia, Religión y Curanderismo. Vol II. Medellín: Editorial Presencia Ltda. UNAL; 1985. p. 9-30.

26. Rocío RI. Organización Panamericana de la salud. Crecer Sanitos. Estrategias, metodologías, e instrumentos para investigar y comprender la salud de los niños indígenas [Internet]. 2003 [citado 10 Nov 2011]. Disponible en: http://www.paho.org/spanish/ad/fch/ca/si-crecersanitos.pdf 
27. Pérez C. Espíritus de Vida y Muerte. Los Miskitu hondureños en época de guerra. Tegucigalpa: Editorial Guaymuras, 2002.

28. Castellanos LG, Flores PF. Representación social del VIH/sida en personas que viven y trabajan en dos centros penitenciarios Mexicanos. Psicología y salud. 2007; 17(01): 33-43.

29. Moscovici S. Notes toward description of social representations. J Experiment Soc Psychol. 1988; 18(3):211-50.

30. Observatoriopic. Observatorio por los derechos y la supervivencia de los pueblos indígenas en Colombia. Embera Chamí [Internet]. 2006 [citado 10 Ene 2012]. Disponible en: http://observatoriopic.org/emberach

Recibido: 22 de febrero de 2012.

Aprobado: 6 de marzo de 2012.

Jaiberth Antonio Cardona Arias. Escuela de Microbiología, Universidad de Antioquia. Calle 67 Número 53-108 oficina 5-103. Colombia. Teléf.: 2198486. Correo electrónico: jaiberthcardona@gmail.com 